\title{
PREDICTION ANALYSIS OF STUDENT LOAN REPAYMENT RATE
}

\author{
Aditya Banerjee, Vikrantsingh R. Bessthakurt, Himanshu Patil, \\ Pratik S. Deshpande and Ali Salem \\ Department of Mechanical and Industrial Engineering, \\ University of Illinois Chicago, United States
}

\begin{abstract}
A student, during his college education, incurs a significant amount of debt. The student's repayment rate can vary according to the institution. There are various factors which depends on different institutes. Factors like features of institution and the earnings of the student after graduating play an important role.

This project tries to realize to what extent these factors predict the student's debt repayment. As a guideline, accuracy of the prediction system, which is assessed using RMSE (Root Mean Squared Error), should be a maximum of 10-11 on the hold-out from the training data.
\end{abstract}

Keywords: Data mining, big data, data analytics, RMSE, Prediction Analysis.

Cite this Article: Aditya Banerjee, Vikrantsingh R. Bessthakurt, Himanshu Patil, Pratik S. Deshpande and Ali Salem, Prediction Analysis of Student Loan Repayment Rate, International Journal of Industrial Engineering Research and Development, 9(1), 2018, pp. 1-6. https://iaeme.com/Home/issue/IJIERD?Volume=9\&Issue=1

\section{INTRODUCTION}

\subsection{Data Mining}

Data mining is the process of sorting through large datasets to identify patterns and establish relationships to solve problems through data analysis. It is the computing process of discovering patterns in large datasets involving methods at the intersection of machine learning, statistics and database systems. It is an essential process where intelligent methods are applied to extract data patterns.

Data mining techniques are used in many research areas, including mathematics, cybernetics, genetics and marketing. In general, the benefits of data mining come from the ability to uncover hidden patterns and relationships in data that can be used to make predictions that impact businesses [21].

\subsection{Feature Selection}

Selecting features plays a very important role in creating a predictive model. Thus, the question arises how to select these features? To accurately select all the features a deep 
knowledge of the problem domain is necessary[ref]. But, fortunately enough it is possible to select features automatically in your data that are most important.

Feature selection also known as variable selection or attribute selection is the process of selecting a subset of relevant features for use in model construction. Though feature selection may appear as a of dimension reduction as both reduce the number of features in the data set. But in the later it is done by creating new combinations of features. Feature selection reduces the redundant features from the data, which otherwise are difficult to predict. This thereby increases the accuracy of the model.

It is desirable to have limited features in data as it reduces complexity and gives better understanding of the domain thereby allowing further manipulation if any less feature reduces the computation time and cost as well [3].

\subsection{Prediction}

Preprocessing steps and definition:

Data Cleaning: Data Cleaning is the process of detecting and correcting (or removing) corrupt or inaccurate records from a record set, table, or database and refers to identifying incomplete, incorrect, inaccurate or irrelevant parts of the data and then replacing, modifying, or deleting the dirty or coarse data4l.

Handling Missing Values: The missing values in this data set are not obviously clear. We can see NaN values instead of NA across different features in the data set. Some unknown values called PrivacySuppressed were also observed. The data shown as 'PrivacySuppressed' in the data set are values which are not reported to protect an individual's privacy. The presence of such values are not good and hinders the predictive ability of the selected features. Such features can be either removed by removing the entire row or converting them into NA and using different methods it can be imputed. Hence, all the values which are NaN or PrivacySuppressed were replaced with Zero 0. By doing this, prediction is allowed and can be reliable.

Scalability: Scalable machine learning algorithms are a class of algorithms which can deal with any amount of data, without consuming tremendous amounts of resources like memory [5].

Robustness: It normally refers to the sensitivity of an estimator with respect to the violation of certain assumptions of the model, especially in finite samples[6].

Interpretability: Interpretability is a relation between formal theories that expresses the possibility of interpreting or translating one into the other.

Accuracy: When a generated machine learning model is tested on previously calibrated data with output, percentage of the output of the model is same as the original output in the test data is measured [7].

Lasso Regression: It stands for Least absolute shrinkage and selection operator. It is a regression analysis method that performs both variable selection and regularization in order to enhance the prediction accuracy and interpretability of the statistical model it produces.

Ridge Regression: It is the most commonly used regression algorithm to approximate an answer for an equation with no unique solution [8].

KNN Algorithm: In pattern recognition, the k-nearest neighbors algorithm is a non-parametric method used for classification and regression [9]. 
Decision Tree: Decision trees are used to build regression or classification models in the form of a tree structure. It breaks down a dataset into smaller and smaller subsets while at the same time an associated decision tree IS incrementally developed [10].

\section{METHODOLOGY}

\subsection{Feature Selection}

In the entire data set, there are several factors which reduces the accuracy of the predictive model, and therefore it was required to choose only those factors which have the highest contribution in the model before proceeding ahead. To fulfill this, Extremely Randomized Trees or Extra-Trees method was chosen.

In extremely randomized trees, randomness is used more extensively in computing the splits when compared to Random Forest Trees. In random forests, a random subset of candidate features is used, but instead of looking for the most discriminative thresholds, Extra-Trees thresholds are drawn at random for each candidate feature and the best of these randomly generated thresholds is picked as the splitting rule. This helps in reducing the variance of the model [11].

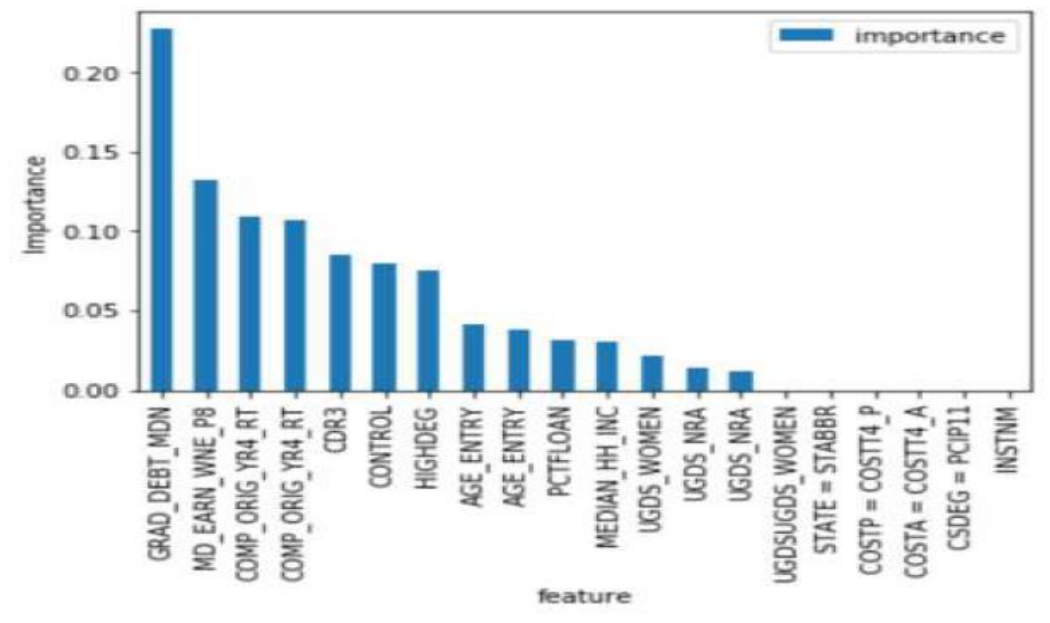

Figure 1 Feature vs Importance

Using this method, we find the predictor variables of our model. The above bar graph was plotted after running the Extra-Tree Regress or function. The top 20 maximum contributing features are obtained, which are as follows:

GRAD DEBT MDN: Median debt for students who have completed.

MD_EARN_WNE_P8:Median earnings of students working and not enrolled 8 years after entry

COMP ORIG_YR4 RT: Percent completed within 4 years at original institution

CDR3: Three-year cohon default rate

CONTROL: Control of institution

HIGHDEG: Highest degree awarded

AGE_ENTRY: Average age of entry

PCTFLOAN: Percent of all undergraduate students receiving a federal student loan

MEDIAN_HH_INC:Median household income

UGDS_NRA: Total share of enrollment of undergraduate degree-seeking students who are non-resident aliens 
UGDS WOMEN: Total share of enrollment of undergraduate degree-seeking students who are women

STABBR: State postcode

COSIT4 P: Average cost of attendance (program-year institutions)

COSIT4_A: Average cost of attendance (academic year institutions)

PCIPI 1: Percentage of degrees awarded in Computer and

Information

INSTNM: Institution name

Table 1 shows the Importance $\%$ of maximum contributing features in the data set.

\subsection{Response Variable}

The response variable of the data set is Repayment rate with values ranging from $0-1$, which is the fraction of students properly repaying their loans. In the data set, it is given under the name of COMPLY_RPY_3YR_RT defined as Three-year repayment rate for completers.

Table 1 Feature Names and Their Importance

\begin{tabular}{|l|c|}
\hline Feature Name & Importance \% \\
\hline GRAD DEBT MDN & 22.98 \\
& 12.62 \\
\hline \multirow{2}{*}{ COMP ORIG YR4 RT } & 11.85 \\
& 10.30 \\
\hline CONTROL & 8.19 \\
\hline HIGHDEG & 8.11 \\
\hline CDR3 & 7.84 \\
\hline AGE_ENTRY & 4.00 \\
\hline MEDIAN HH INC & 2.95 \\
\hline PCTFLOAN & 2.93 \\
\hline UGDS WOMEN & 2.08 \\
\hline LIGDS NRA & 1.21 \\
\hline UGDS_NRA & 1.12 \\
\hline
\end{tabular}

\subsection{Splitting into Train-Validation-Test sets}

After analyzing the features and removing the randomly missing values, the dataset is split into training, validation and test set. The test train split has been taken as $20-80 \%$ of the dataset.

\subsection{Modeling}

Models like linear regression, Lasso regression, Decision Tree Regression, and Ridge regression has been performed on the data and the predictive score is compared.

Model is built on the training set and then applied on the validation set to calculate the Rsquare score, Mean Absolute Error and Mean Squared Error. This would help in validating how well the model is predicting, and also to check if the model is working properly and isn't over-fitting the training set.

\section{CONCLUSIONS}

The Mean Squared error of the entire model came out to be around 0.03, which shows that the model has performed very well and didn't overfit the training set. 
Fig. 2 and Table 11, shows that the Linear and Ridge Regression models, are giving us highest R-squared scores amongst all the four regression models.

Table 2 R2 Scores of Different Models

\begin{tabular}{|l|c|}
\hline \multicolumn{1}{|c|}{ Model Type } & R2 Score \\
\hline Linear Regression & $57.71 \%$ \\
\hline Decision Tree & $53.47 \%$ \\
\hline Lasso Regression & $45.92 \%$ \\
\hline Ridge Regression & $57.71 \%$ \\
\hline
\end{tabular}

However, after computing the four regression models, KNN Regression algorithm was implemented into the model, to analyze whether the score of the model can be improved or not.

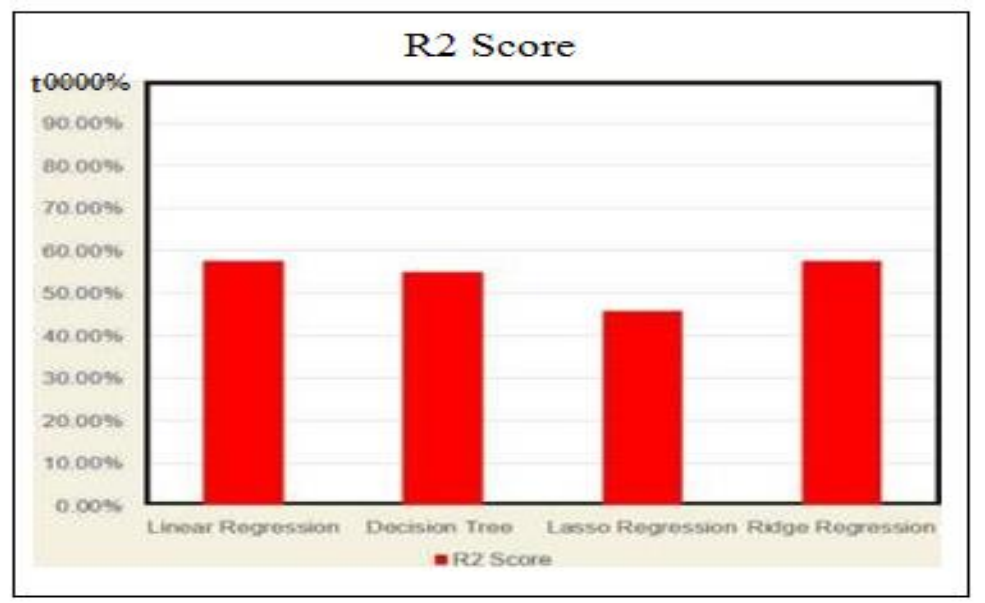

Figure 2 Regression models vs R2 Score

Fig. 3 exhibits that with number of neighbors as $27, \mathrm{KNN}$ regressor estimator gives us a score of $61.45 \%$, thus further improving the predictive score of the model.

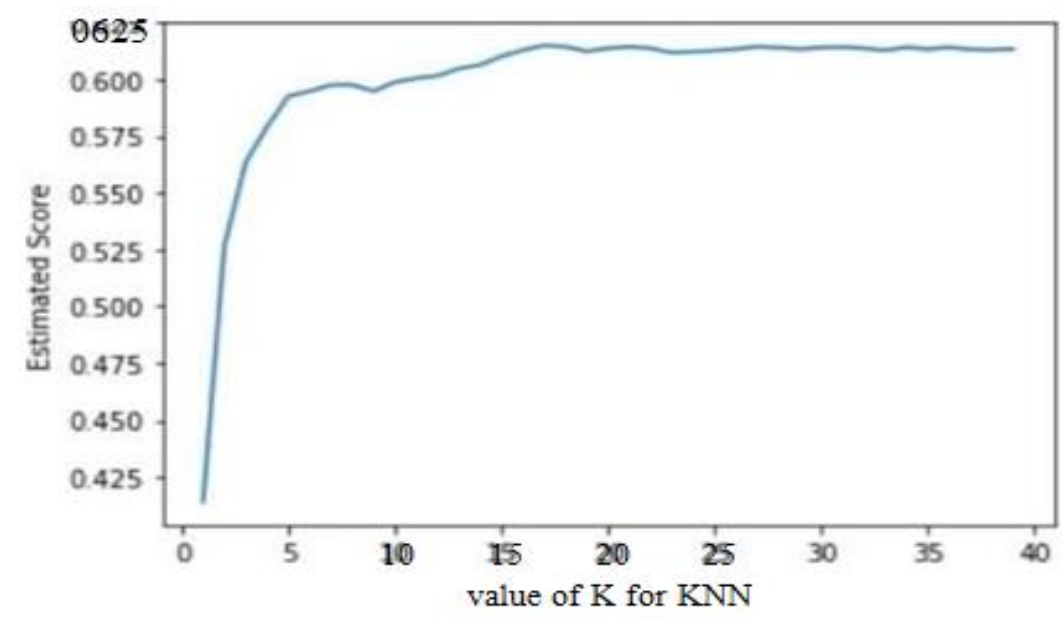

Figure $3 \mathrm{~K}$ value of neighbors vs the Estimated Score

\section{ACKNOWLEDGMENT}

We would like to show our gratitude to Prof. Dr. Ashkan Sharabiani for sharing his wisdom with us during the course of this project. We are also immensely grateful to Sourabh Parime 
for his comments and guidance that greatly improved this project. We are also thankful to moderators and members of Stack Exchange, Analytics Vidhya, and Data Camp.

\section{REFERENCES}

[1] Paudel, Barun. (2017), Project Report-Student Loan Repayment Prediction, 10.13140/RG.2.2.17610.47046

[2] Rouse, Margaret. Definition data mining. Retrieved from http://searchsqlserve".techtarget.com/definition/data-mining

[3] Isabelle Guyon and Andre Elisseeff, "An Introduction to Variable and Feature Selection", Journal of Machine Learning Research, VO1.3, 2003, pp.1157-1182, ISSN: 1533-7928

[4] Data cleansing. Retrieved from https://en.wikipedia.org

[5] Rishi, Deepak. (2015, April 9). What is Scalable Machine Learning? What are some good resources to learn about it? Retrieved from https://www.quora.com

[6] What is the difference between robustness and consistency in econometrics? Retrieved from https://www.quora.com

[7] Vobugari, Neelima. (2017, December 8). What is accuracy in machine learning? Retrieved from https://www.quora.com

[8] Padmanabha, Akshay. Ridge Regression. Retrieved from https://brilliant.org

[9] N. S. Altman (1992) "An Introduction to Kernel and Nearest-Neighbor Nonparametric Regression", The American Statistician, 46(3), 175-185, DOI: www.doi.org/10.1080/00031305.1992.10475879

[10] Pedregosa, Fabian et al., "Scikit-learn: Machine Learning in Python", Journal of Machine Learning Research, 2011, Vol.12, pp. 2825-2830

[11] Florin, Schimbinschi. (2015, December 5). Difference between Random Forest and Extremely Randomized Trees. Retrieved from https://stats.stackexchange.com/q/185840 